\title{
Influence du tégument des graines mûres de Vicia faba sur le développement larvaire de Callosobruchus maculatus
}

\author{
A. Boughdad, Y. Gillon \& C. Gagnepain \\ Laboratoire d'Entomologie Bât. 446, Université Paris-Sud, F-91405 Orsay cedex, France
}

Mots Clés: Callosobruchus maculatus, graines, Vicia faba, tégument, barrière chimique, tanins condensés

Keywords: Callosobruchus maculatus, pulses, Vicia faba, husk, chemical barrier, condensed tannins

\begin{abstract}
Des élevages expérimentaux de Callosobruchus maculatus (F.) (Coleoptera, Bruchidae) sur les graines de Vicia faba décortiquées ou non, ou sur des pastilles faites des mêmes graines, ont permis de mettre en évidence une barrière empêchant le développement de la bruche.

Avec les graines entières, toutes les larves meurent au premier stade enfoncées dans le tégument; alors qu'elles se développent normalement dans les graines décortiquées.

Avec des pastilles de broyats de graines non décortiquées, la majorité des larves meurt avant le terme de leur développement; la plus forte mortalité est observée au quatrième stade larvaire. Les larves, qui survivent, accusent une augmentation de leur durée de développement par rapport à celles qui se nourrissent aux dépens des pastilles faites de graines sans tégument.
\end{abstract}

\section{Introduction}

Lors du stockage, les graines de fèves font partie des hôtes potentiels de $C$. maculatus (Boughdad, 1982). Les larves de cette bruche se développent aux dépens des réserves nutritives localisées au niveau des cotylédons et du germe. Pour atteindre ces organes, la larve néonate doit traverser le tégument de la graine. Mais le succès du développement larvaire varie selon les variétés attaquées (El Sawaf, 1956; Labeyrie, 1962; Hariri, 1981; Boughdad, 1981).

Des expérimentations, menées sur $C$. chinensis par Podoler \& Applebaum (1968), mettent en évidence la résistance de certaines variétés de graines de fèves en relation avec la dureté et l'épaisseur du tégument. D'autres chercheurs, cependant, imputent ce phénomène à l'action des saponines présentes dans les graines (Applebaum et al., 1969). En utilisant des tanins condensés extraits de tégument de certaines variétés de fèves, Griffiths \& Jones (1977) ont montré, in vitro, une réduction de la solubilité des protéines cotylédonaires d'une part, et une moindre activité des enzymes digestives d'autre part.
Par ailleurs, Martin-Tanguy et al. (1977) ont observé une réduction de la croissance pondérale et de la reproduction des canards élevés à partir de graines de fèves, riches en tanins condensés.

Pour notre part, après plusieurs tentatives infructueuses d'élevage de $C$. maculatus aux dépens des graines de fèves, nous nous sommes demandés si le tégument de ces fèves n'était pas responsable de ces échecs. Ce tégument ne constituait-il pas un obstacle mécanique s'opposant à la pénétration des larves nouveau-nées, ou se comportait-il aussi comme une barrière chimique, empêchant ces larves d'atteindre les organes de réserves en provoquant leur mort?

Ce travail s'attache à préciser la nature et l'efficacité du tégument, comme barrière empêchant la consommation de $V$. faba par C. maculatus.

\section{Matériel et méthodes}

La souche de C. maculatus provient de l'I.B.E.A.S. de Tours (France), où elle était élevée sur les graines de Vigna radiata. Dans notre 
laboratoire, l'élevage a été poursuivi pendant plus de 10 générations successives sur graines de Vigna unguiculata.

Les graines de $V$. faba utilisées pour les expériences sont de la variété 'Géante Portugaise'. Elles sont conservées au congélateur à $-18^{\circ} \mathrm{C}$.

Les conditions de toutes les expériences sont: photopériode: L12:D12; thermopériode: photophase $30 \pm 1^{\circ} \mathrm{C}$, scotophase $21 \pm 1^{\circ} \mathrm{C}$; humidité relative: $70 \%$.

Des couples, dès leur émergence des graines de $V$. unguiculata, sont mis à pondre sur des graines de fèves décortiquées ou non, ou sur des pastilles, faites respectivement des mêmes graines, en équilibre hydrique. Les pastilles, constituées de la farine des graines broyées (granulométrie maximale $=0.5 \mathrm{~mm}$ ), sont confectionnées au moyen d'une presse de type Prolabo. Elles pèsent $1 \mathrm{~g}$ chacune, et, ont un diamètre de $0.8 \mathrm{~cm}$ et une épaisseur de $0.5 \mathrm{~cm}$.

Après $24 \mathrm{~h}$, les bruches sont retirées et les oeufs avec leurs supports sont replacés dans lés conditions d'élevage précitées. A l'éciosion, quatre lots distincts de 100 larves chacun sont constitués. Le nombre de larves par graine ou pastille est fixé de telle sorte qu'elles n'entrent pas en compétition entre elles (Mitchell, 1975).

Les bruches émergeant de chaque substrat alimentaire sont recensées, leurs dates d'émergence, leurs sexes ainsi que leurs poids frais sont relevés. Quand tous les adultes ont émergé ( 3 mois après la ponte), le nombre et le stade des bruches mortes dans chaque aliment sont notés. Les stades larvaires ont été caractérisés en effectuant les mensurations de la capsule céphalique.

\section{Résultats}

Les valeurs des critères biologiques de C. maculatus sont déterminées pour chaque type d'aliments. Nous avons analysé successivement les taux de mortalité, les durées de développement postembryonnaire et les poids à l'émergence.

Taux de mortalité. C. maculatus présente des mortalités variant selon le stade du développement et le substrat nutritif larvaire (Fig. 1).

Avec les graines entières, aucun insecte ne dépasse le premier stade larvaire; toutes les larves meurent enfoncées dans le tégument des graines
(Fig, 1, A). La profondeur des forages est in moyenne de $0,15 \mathrm{~mm}$ (cas de $85 \%$ de larves nouveau-nées). Seulement $15 \%$ des larves du premier stade dépassent cette profondeur. Le maximum atteint est de $0.23 \mathrm{~mm}$. Cette dernière valeur correspònd à l'épaisseur moyenne des téguments des graines utilisées (nombre de téguments mesurés $=50$, sem $= \pm 0.03 \mathrm{~mm}$ ).

Les graines décortiquées permettent à $70 \%$ des bruches d'accomplir leur développement complet. Le reste de la population ( $22 \%$ ) connaît une mortalité variable selon le stade de développement (Fig. 1, B). C'est le quatrième stade larvaire qui enregistre, le plus fort taux de mortalité $(11 \%)$; les mortalités des autres stades sont faibles (Fig. 1, B).

Dans les deux catégories de pastilles (avec ou sans tégument) la bruche peut parvenir au terme de son développement. Cependant, les effectifs des descendants obtenus ainsi que la mortalité varient selon que les pastilles renferment de la poudre de tégument ou non. Les pastilles dépourvues de tégument permettent d'obtenir une proportion d'adultes très voisine de celle observée avec les graines décortiquées. Là aussi, c'est le quatrième stade larvaire qui accuse une forte motalité $(13 \%)$ par rapport aux autres stades dans le même aliment (Fig. 1, D). Dans le mélange farine avec tégument, $62 \%$ de la population mise en élevage meurent avant d'atteindre le terme de leur développement. Ici les plus fortes mortalités surviennent au cours des premier et quatrième stades larvaires (Fig. 1, C). Enfin contrairement à ce qu'il advient avec les aliments dépourvus de tégument, où le nombre de femelles est légèrement supérieur à celui des mâles, la fréquence des mâles est ici supérieure à 0.5 .

Durée du développement post-embryonnaire. Elle varie selon le sexe et les substrats nutritifs larvaires (Fig. 2). Avec un même aliment, le développement des mâles est plus rapide que celui des femelles. Les mâles commencent à émerger 1 à 2 jours avant les femelles (Fig. 2).

Pour un même sexe, les individus s'étant développés aux dépens des graines décortiquées présentent une durée de développement postembryonnaire plus courte que celle des animaux issus des pastilles faites avec les mêmes graines décortiquées ou non. Lémergence commence 5 jours avant celle des insectes provenant des mêmes graines décortiquées mais broyées (Fig. 2).

Quand la farine des graines est additionnée de 

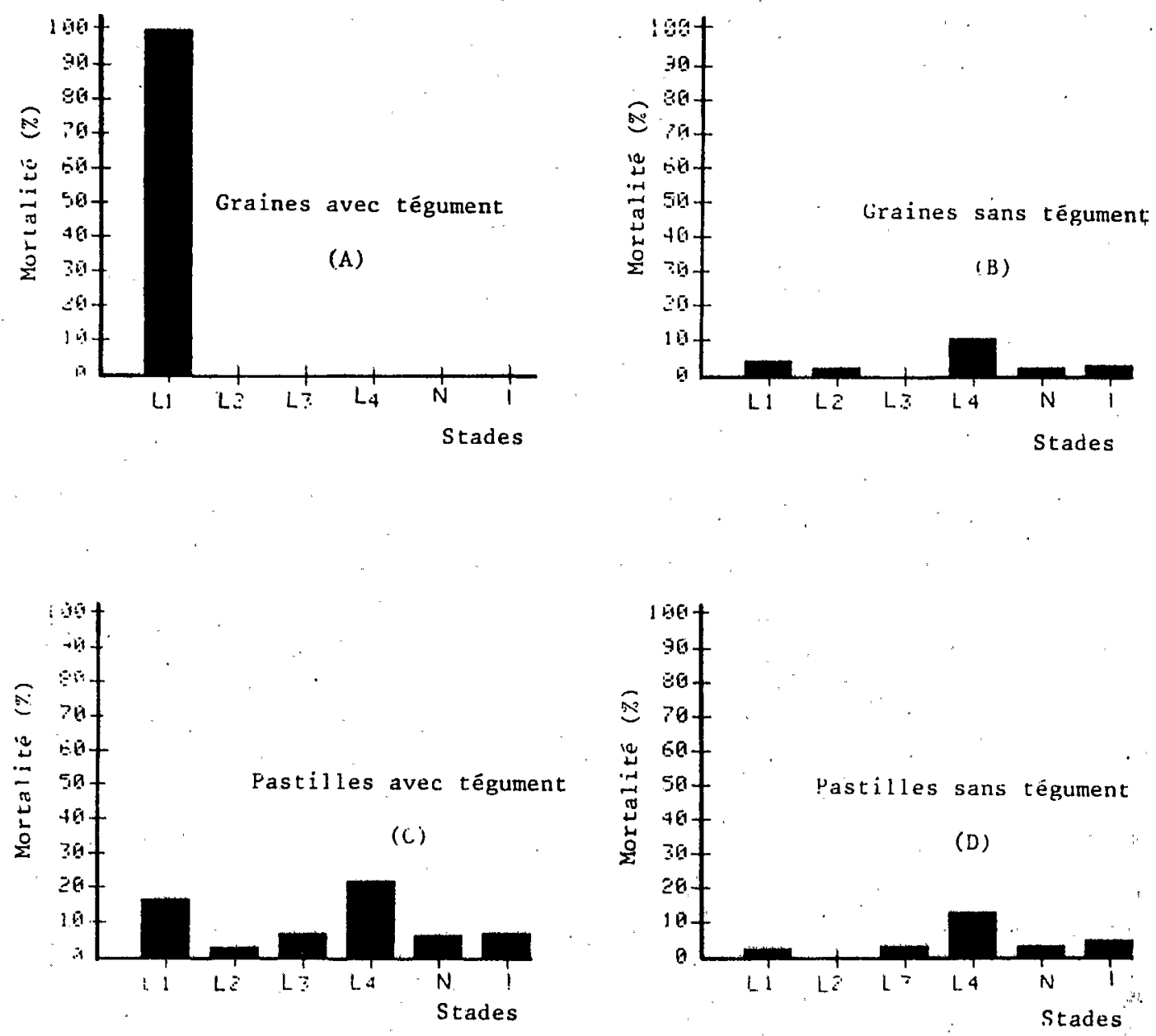

Fig. 1. Mortalité de C. maculatus à l'intérieur des substrats nutritifs à base des graines de $V$. faba (L: stades larvaires, N: nymphe, I: imago).

Mortality of $C$. maculatus reared on $V$. faba seeds and on pellets made from V. faba flour (L: larval instars, N: nymph, I: imago).

téguments broyés, le développement des bruches est plus étale (Fig. 2). En effet, dans les pastilles sans tégument, les émergences s'échelonnent sur 13 et 14 jours respectivement pour les mâles et pour les femelles; alors qu'elles s'étalent sur 18 et 19 jours pour leurs homologues respectifs dans les pastilles munies de tégument (Fig. 2). De plus, avec les broyats sans tégument, $50 \%$ d'adultes émergent le quatrième ou le cinquième jour du début des émergences; tandis qu'avec la farine renfermant des téguments, la même proportion n'est atteinte que le huitième ou le neuvième jour après les premières émergences (Fig. 2).

Poids à l'émergence. Pour un même sexe, le poids moyen est. voisin $(2,8 \pm 0,6 \mathrm{mg}$ pour les mâles et $3.6 \pm 0,8 \mathrm{mg}$ pour les femelles), quel que soit le milieu du développement larvaire. Les femelles pèsent plus lourd que les mâles. Cette homogénéité des poids à l'émergence est obtenue avec des vitesses de développement variées: la plus rapide correspond aux graines décortiquées et la plus lente aux pastilles avec tégument.

\section{Discussion}

Le tégumient des graines de fèves testées constitue donc pour C. maculatus une barrière infranchissable, qui empêche le développement larvaire de la 

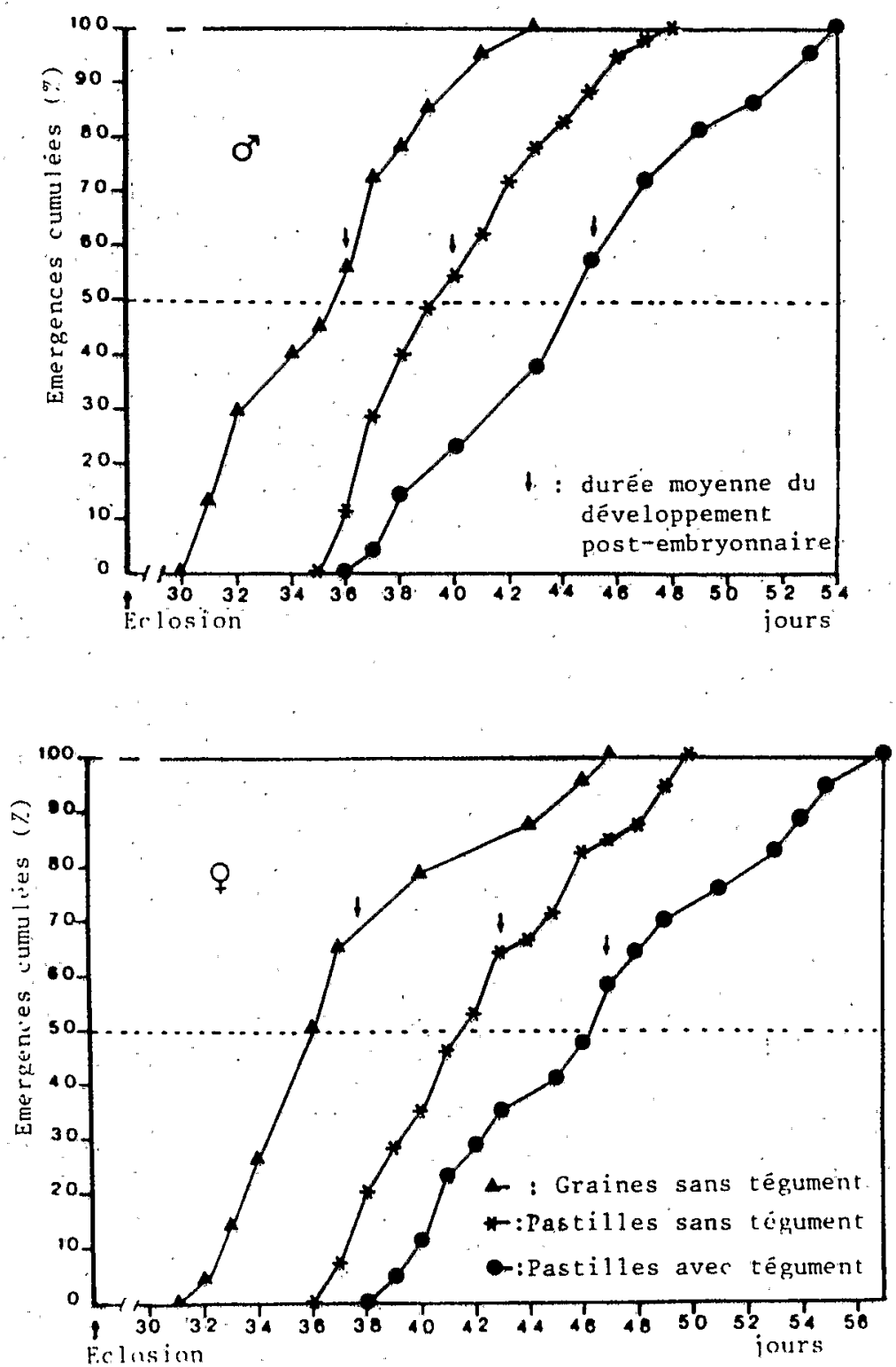

Fig. 2. Emergences d'adultes de $C$. maculatus de différents substrats nutritifs à base des graines de $V \cdot \dot{f a b a}$. Emergence of adults of $C$. maculatus from different nutritive substrates made with $V$. faba seeds:

bruche. Cette barrière est essentiellement chimique et liée à la présence des tanins condensés dans le tégument des fèves (Boughdad et al., 1986). Un effet toxique des tanins condensés commerciaux a été mis en évidence chez la même bruche par Janzen et al. (1977).

La morțalité de la majorité des larves néonates, au niveau des premières épaisseurs du tégument, laisse supposer que ces composés phénoliques sont concentrés dans les couches externes de l'enveloppe, comme l'ont signalé Zimmerman et al. (1967).

Ladjonction du tégument, aux broyats des cotylédons de fèves, provoque la mortalité d'environ $49 \%$ de l'effectif initial et retarde le développement des survivants. La forte mortalité du quatrième stade larvaire pourrait résulter $\mathrm{d}^{\prime} u n$ effet cumulatif comme le suggèrent Stamopoulos \& Huignard (1980) chez Acanthoscelides obtectus sur 
les graines de Phaseolus vulgaris. Une différence de sensibilité des stades consommateurs du broyat est également envisageable. Enfin la modification de la texture de l'aliment pourrait se traduire par une mauvaise alimentation larvaire, dont l'effet s'amplifie au cours du développement des larves. Ceci pourrait expliquer la mortalité élevée du quatrième stade larvaire. Stamopoulos \& Desroches (1981) ont fait une remarque similaire chez $A$. obtectus élevé sur de la poudre du haricot.

Cette étude met en évidence l'efficacité du tégument de $V$. faba contre l'attaque des bruches. Cette barrière est de nature chimique. L'effet négatif des tanins condensés extraits du tégument des mêmes graines sur le développement de $C$. maculatus a été démontré (loc. cit.). Il reste à préciser le mode d'action de ces substances sur les différents stades de développement de la bruche.

\section{Summary}

Effect of the ripe seed husks of Vicia faba on the larval development of Callosobruchus maculatus

Experimental rearing of Callosobruchus maculatus was conducted on Vicia faba beans with or without their tegument, or on flour of the same beans, compressed as pellets.

These experiments demonstrated a barrier preventing bruchid development upon the broad bean seeds.

On the whole beans, all the larvae died at the first instar, within the husk, whereas they normally thrive on decorticated beans.

In pellets made with whole bean flour, the majority of larvae died before reaching their complete development. The highest mortality occurred at the fourth larval instar. The surviving bruchids had a longer development period than those feeding upon pellets made with decorticated beans flour.

We conclude that the barrier preventing southern cowpea weevil development on horse beans is chemical. This barrier consists of the condensed tannins present in $V . f a b a$ tegument. Isolation, identification and studies of the biological effect of those phenolic compounds are being undertaken (Boughdad et al., 1986).

\section{References}

Applebaum, S. W., S. Marco \& Y. Birk, 1969. Saponins as possible factors of resistance of legume to the attack of insects. J. Agric. food Chem. 17: 618-622.

Boughdad, A., 1981. Influence des composés primaires de certaines graines de légumineuses alimentaires sur divers critères biologiques de Callosobruchts maculatus (F.) (Coleoptera, Bruchidae). DEA-Entomologie, Paris VI. pp. 39.

Boughdad, A., 1982. Déprédateurs des denrées entreposées au Maroc. In: Colloque International sur les denrées stockées. 10-11 mai 1979, Rabat, OEPP: 17-23.

Boughdad, A., Y. Gillon \& C. Gagnepain, 1986. Influence des tanins condensés du tégument de fèves (Vicia faba) sur le développement larvaire de Callosobruchus maculatus. Entomol. exp. appl. 42 (sous presse).

El Sawaf, S. K., 1956. Some factors affecting the longevity, oviposition and rate of development in the southern cowpea weevil, Callosobruchus maculatus (F.). Bull. Soc. Entomol. Egypte 11: 29-95.

Griffiths, D. W. \& D. I. H. Jones, 1977. Cellulase inhibition by tannins in the testa of field beans (Vicia faba). J. Sci. Food Agric. 23: 983-989.

Hariri, G., 1981. Distribution and importance of bruchid attacks on different species of pulses consumed in the Near-Est. In: V. Labeyrie (ed.), The Ecology of Bruchids attacking Legumes (pulses). Dr. W. Junk Publishers, The Hague: pp. $515-521$.

Janzen, D. H., H. Juster \& E. Bell, 1977. Toxicity of secondary compounds to the seed-eating larvae of the bruchid beetle Callosobruchus maculatus. Phytochemistry 16: 223-227.

Labeyrie, V., A. Hoffmann \& A. S. Balachovsky, 1962. Famille de Bruchidae. In: Masson \& Cie. (eds), Entomologie appliquée à l'Agriculture. Tome I, Coléoptères, Vol. 1. Paris: pp. 434-494.

Martin-Tanguy, J., J. Guillaume \& A. Kossa, 1977. Condensed tannins in horse bean seeds: Chemical structure and apparent effects on poultry. J. Sci. Food Agric. 28: 757-765.

Mitchell, R., 1975. The evolution of oviposition tactics in the bean weevil, Callosobruchus maculatus (F.). Ecology 56: 696-702.

Podoler, H. \& S. W. Applebaum, 1968. Physiological aspects of host specificity in the bruchidae. V. Varietal differences in the resistance of Vicia faba to Callosobruchius chinensis L. J. Stored Prod. Res. 7: 97-105.

Stamopoulos, D. \& P. Desroches, 1981. Influence of the tegument of Phaseolus vulgaris seeds and of larval density on the development of Acanthoscelides obtectus Say. In: V. Labeyrie (ed.), The Ecology of Bruchids attacking Legumes (pulses). Dr. W. Junk Publishers, The Hague: pp. 165-174.

Stamopoulos, D. \& J. Huignard, 1980. Linfluence des diverses parties de la graine de haricot (Phaseolus vulgaris) sur le développement des larves d'Acanthoscelides obtectus (Coléoptère, Bruchidae). Entomol. exp. appl. 28: 38-46.

Zimmermann, G., S. Weissmann \& S. Yannai, 1967. The distribution of proteins, lysine and methionine, and antitryptic activity in the cotyledons of some leguminous seeds. J. Food Sci. 32: 129-130.

Accepted: May 16, 1986. 